\title{
Application of the ground penetrating radar to detect weapons caches and unexploded ordnance: laboratory experiments
}

\author{
Hussein Khalefa Chlaib ${ }^{1}$, Wathiq Abdulnaby ${ }^{2}$, Najah $\mathrm{Abd}^{3}$ \\ ${ }^{I}$ (Department of Physics, College of Sciencel Thi-Qar University, Iraq) \\ 2 (Department of Geology, College of Science/ University of Basrah, Iraq) \\ 3 (Department of Geology, College of Science/ University of Baghdad, Iraq)
}

\begin{abstract}
For the security reasons, buried weapons need to be detected. This study explains laboratory experiments of using the Ground Penetrating Radar (GPR) to study the feasibility and ability of this technique to detect these subsurface caches and calculate the target depth and dimensions. Iron, plastic, wooden boxes filled with metallic materials, and empty plastic box buried in the sand box had been used as examples of the weapons caches. The GPR system had been run over these materials with $1500 \mathrm{MHz}$ GSSI antenna. The GPR data indicate that all the four cases are predictable and investigated by this non-destructive geophysical tool. Also, the distinctions between the different weapons containers and the filled or empty boxes are easy to be investigated by the GPR system.
\end{abstract}

Keywords: Ground Penetrating Radar, Antenna, Dielectric constant, Caches, Electromagnetic waves.

\section{Introduction}

The terrorist groups have a lot of different weapons such as, guns, rifles and shooters, unexploded ordnance (UXO), Improvised Explosive Devices, and explosive stuck devices, and explosive belts. Also they use the houses in the popular areas to store and as caches of their weapons and other devices. They usually dig holes inside the houses and rooms or in the houses yards, under the concrete slabs or flagstones and they but these weapons then they buried the holes. Most of times the police and army cannot find these caches because they are located under vary depths, and under the flagstones or the concrete. Also, they are difficult to detect because of the containers materials nature.

In this experimental, we try to study the ability, capability, sensitivity, and effectiveness of the GPR to detect and locate the weapons caches and unexploded ordnance, and buried explosive devices. Additionally, we try to know the approximate dimensions and the depth of these materials and estimate the kinds of the containers materials. GPR are described as noninvasive and safe method used a wide range of electromagnetic waves to detect and image subsurface targets and mediums without surface disruption and ground excavation [1]. The first description of the electromagnetic signals using to locate subsurface targets and buried objects was in Germany in 1910 [2]. In the world war II, GPR had been used to detect the buried airplanes and military ships [3]. In the second half of 1960s during Vietnam's war, GPR had been used by US army to detect the subsurface tunnels [3]. From the 1970s to the present day, GPR application developed widely and it involves in tens of the applications [2].

GPR has been used in historical and archaeological applications since the 1970s [4, 5]. Hydrological studies are one of the important GPR application Fields $[6,7]$. GPR technique is well accepted tool to study the highway, roads, and bridge deck evaluations $[8,9]$. Also, GPR is applicable in the geotechnical, geological, engineering, and environmental fields $[10,11,12,13]$.

\section{The GPR Applications on Weapons and Unexploded Ordnance}

There are no scientific researches or studies to apply the GPR technique to detect and investigate the subsurface stores and underground weapons and unexploded ordnance. Some military applications use electromagnetic waves properties to detect military small part, the targets located under shallow depth and for small areas. These electromagnetic devices like an iron sensors give a specific sound when they detect a metallic object. Also these devices do not give at the exact depth, size, and dimensions of the target. In this study we did laboratory experiments to see the effectiveness and feasibility of the GPR technique and its applications on weapons and unexploded ordnance detection.

\section{GPR Fundamental Principles}

GPR is an efficient technique for imaging shallow targets. Recently GPR is one of the preferring geophysical tools due to:

- The survey and measurements are relatively easy to obtain.

- Its high vertical and horizontal resolution. 
- The light weight and mobility of the equipments.

- It obtains a continuous profiling for long distance.

- Non-invasive technique and does not need intrusive ground contact.

- The survey can be made in any terrain.

- Wide range of multi- frequency antennas (16 MHz to $2 \mathrm{GHz}$ ) can be selected.

- High sensitive to water.

GPR antenna has two parts, transmitter and receiver, the transmitting antenna generate electromagnetic pulse. The pulse spreads and transmits into the ground. The receiver picked-up the bock scatter and reflected waves that encounter a discontinuity in the dielectric properties (e.g. void, pipe, new layer). The remaining electromagnetic waves keep going and continue to deeper subsurface levels (Fig. 1).

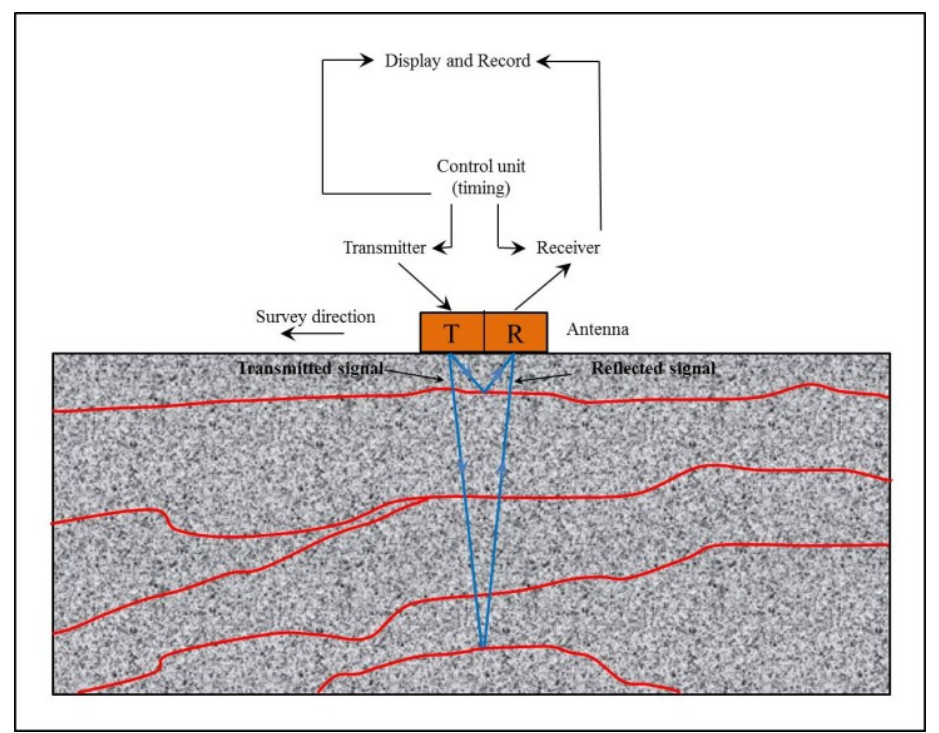

Figure 1: Principles of GPR.

The speed of the transmitted GPR signals are various depend on the medium's electromagnetic properties that travel through. Medium's Relative dielectric permittivity (dielectric constant) is one of the important dielectric parameters that control the GPR signal velocity. Dielectric constant $\varepsilon$ r, is defined as:

$\varepsilon_{\mathrm{r}}=\varepsilon / \varepsilon_{0}$

Where:

$\varepsilon$ : The medium permittivity

$\varepsilon_{0}$ : The vacuum permittivity $\left(8.89 * 10^{-12} \mathrm{~F} / \mathrm{m}\right)[14]$.

The dielectric constant of the material is depending on many complex factors, the important effective factors are water content and presence of the clay minerals (air has the lowest dielectric constant $=1$ and water has the highest value $=81$, therefore water control the dielectric permittivity of the medium). GPR signal velocity $v(\mathrm{~m} / \mathrm{ns})$, can calculated as below:

$v=c / \sqrt{ } \varepsilon_{\mathrm{r}}$

Where:

$c$ : The speed of light in vacuum $\left(3 * 10^{8} \mathrm{~m} / \mathrm{s}\right)$ or $(0.3 \mathrm{~m} / \mathrm{ns})$.

$\varepsilon_{\mathrm{r}}$ : The dielectric constant of the terrain.

Also $v$ can calculate if the anomaly's depth and wave travel time are known, it equal:

$v=2 * D / \Delta t$

Where

$D$ : the depth to the target.

$t$ : is the travel time.

Note the number 2 refers to the two travel time that the signal need to travel from the transmitter, reach the target and reflected back to the receiver.

$D=v^{*} t / 2$

$D=c * t / 2 \sqrt{ } \varepsilon_{\mathrm{r}}$

The GPR signal reflective contrast or strength, at interfaces of materials of different lithology or different dielectric constant, is called the reflection coefficient $\mathrm{R}$, and it determines by using the following equation:

$R=\sqrt{ } \varepsilon_{1}-\sqrt{\varepsilon_{2}} / \sqrt{ } \varepsilon_{1}+\sqrt{ } \varepsilon_{2}$ 
$\varepsilon_{1}$ : The dielectric constant of the upper media.

$\varepsilon_{2}$ : The dielectric constant of the lower media.

The GPR penetrating depth is depend on, antenna's frequency and subsurface materials electrical properties. Surveys conducted with lower frequency EM waves will have less attenuation of the signal and thus deeper penetration, but lower resolution. Many factors reduce the penetrating depth such as, water content and presence of clay materials because they increase the electrical conductivity [14].

\section{Data Collection}

This laboratory work was conducted in the geophysical laboratories at Applied Science Department, University of Arkansas at Little Rock (UALR), USA. To facilitate the GPR laboratory experiment, a wooden box having dimensions (outside) of $135 \mathrm{~cm}$ length, $59 \mathrm{~cm}$ high, and $53 \mathrm{~cm}$ wide (Fig. 2), an iron $(20 \mathrm{~cm}$ length, $13 \mathrm{~cm}$ wide, and $7 \mathrm{~cm}$ high ), plastic (22 cm length, $18 \mathrm{~cm}$ wide, and $10.5 \mathrm{~cm}$ high $)$, and wooden $(19.5 \mathrm{~cm}$ length, $15 \mathrm{~cm}$ wide, and $9 \mathrm{~cm}$ high ) boxes filled with iron and copper materials was used as a subsurface targets (weapons and unexploded ordnance) as showed in Fig. 3.

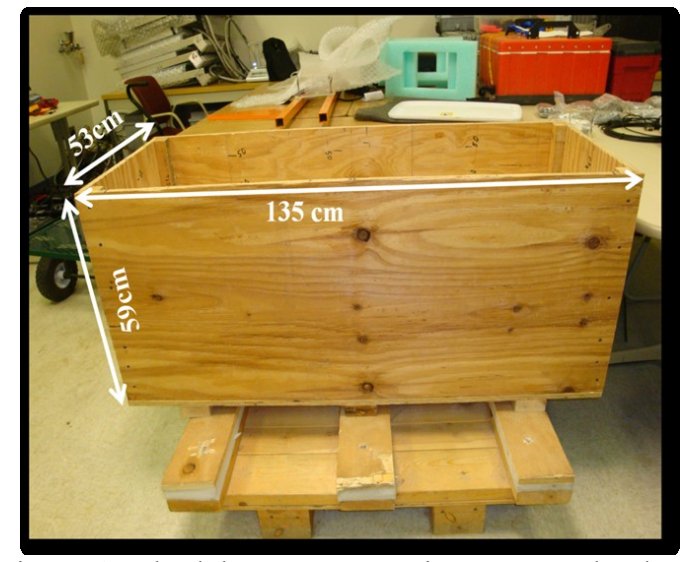

Figure 2: The laboratory experiments wooden box.
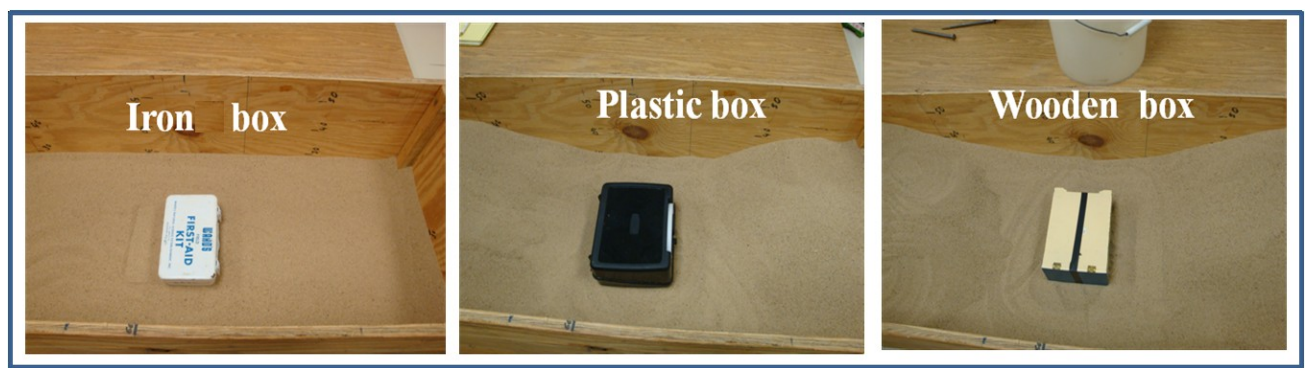

Figure 3: An iron (20 cm length, $13 \mathrm{~cm}$ wide, and $7 \mathrm{~cm}$ high), plastic $(22 \mathrm{~cm}$ length, $18 \mathrm{~cm}$ wide, and $10.5 \mathrm{~cm}$ high), and wooden ( $19.5 \mathrm{~cm}$ length, $15 \mathrm{~cm}$ wide, and $9 \mathrm{~cm}$ high) boxes filled with iron and copper materials were used as a subsurface targets (weapons and unexploded ordnance).

The data for this experiment was collected by SIR-3000 Geophysical Survey System Inc. (GSSI) [15], with a $1.5 \mathrm{GHz}$ central frequency monostatic antenna (Fig. 4). Each $7 \mathrm{~ns}$ scan was sampled at discrete 16 bits per sample and 1024 samples per scan. On average, profiles consist of about ((iron) 1549, (plastic) 1977, (wooden) 1857, and (empty plastic) 2095) scans, data were bandpass filtered using (system default) high-pass and low-pass filters of $75 \mathrm{MHz}$ and $700 \mathrm{MHz}$, respectively. The three metallic material filled (iron, plastic, and wooden) boxes and empty plastic box buried in the sand at the depth $25 \mathrm{~cm}$ (from the top of sand to the bottom of the boxes). Scan started $15 \mathrm{~cm}$ away from the sand box left edge (to ignore the side reflections coming from the wood box).

For each buried box, two antenna configurations (polarizations) were examined to observe the effect of the survey direction in relation to the antenna radiation pattern. The two antenna's polarizations (one perpendicular on the box sand long axis, while the second one is parallel to the long axis) are shown in the Fig. 5. Steven's Soil Sensor was used to measure the sand dielectric constant, it was (2.6)., this value is so important to calculate the target's depth [16]. 


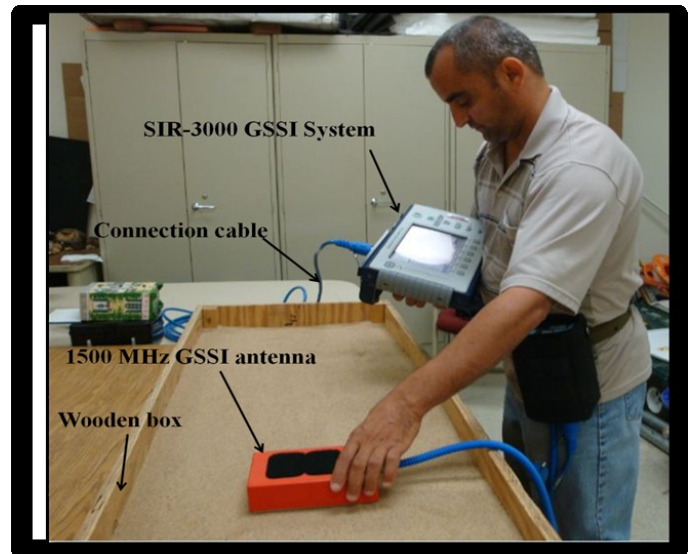

Figure 4 : The GPR system that used in the laboratory experiment.

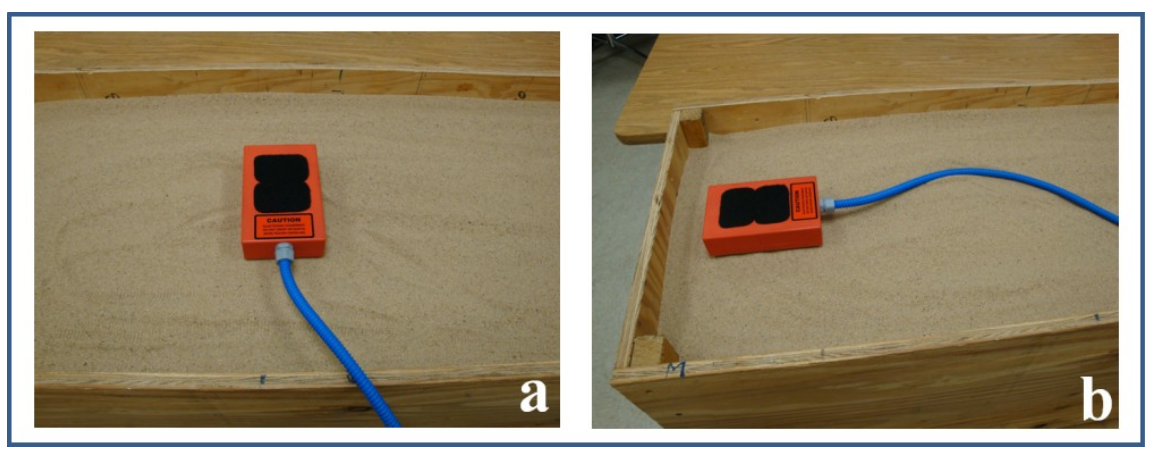

Figure 5: Antenna's radiation pattern (polarization). a, perpendicular on the box sand long axis, b, parallel to the long axis).

\section{Data Processing}

The data processing can modify the GPR row data for easy and clear visualization and more accurate interpretation. All the GPR data were post-processed by using RADAN 7 GSSI software [17, 18]. Zero time process applied to adjust the zero-time with zero-depth to have the accurate depth of the targets; therefore, we will remove any time offset due to the system recording before the interpretation (Fig. 6).

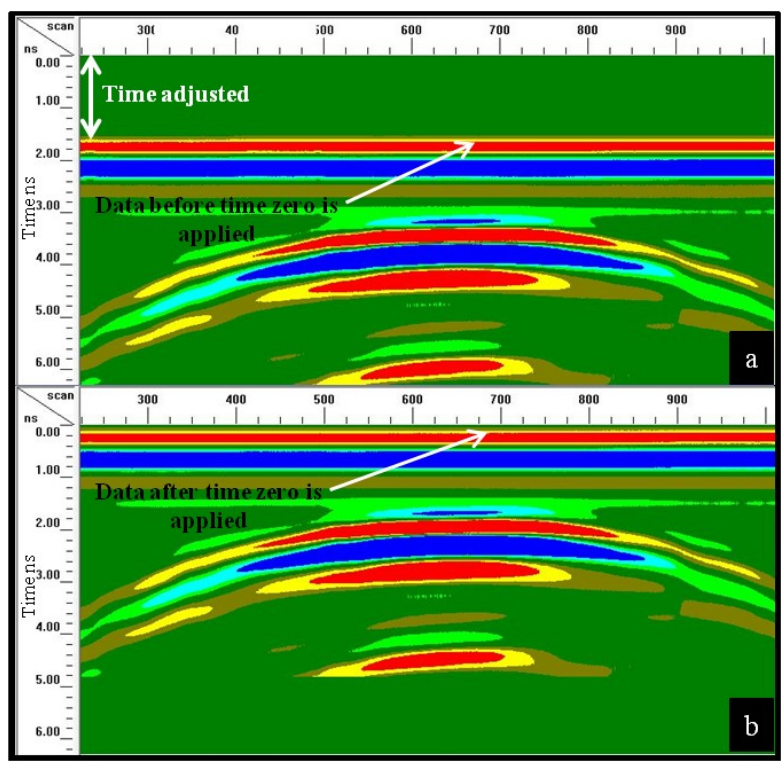

Figure 6: Portion of GPR data (1500 MHz antenna) of the iron box buried in the sand. a, before time zero process, $\mathrm{b}$, after time zero process. 
The background removal is one of the most common processes. It uses to remove the noise and unwanted reflections that appear as a horizontal line in the GPR profile data. These desirable reflections are caused by antenna ringing and the multiple reflections between the antenna and the ground surface. The deeper structure may be completely masked by the previous reflections, especially if the second one is strong and not properly removed. Fig. 7 shows the background removal process for the GPR data collected with $1500 \mathrm{MHz}$ antenna of the wooden box filled with ironic and copper material.

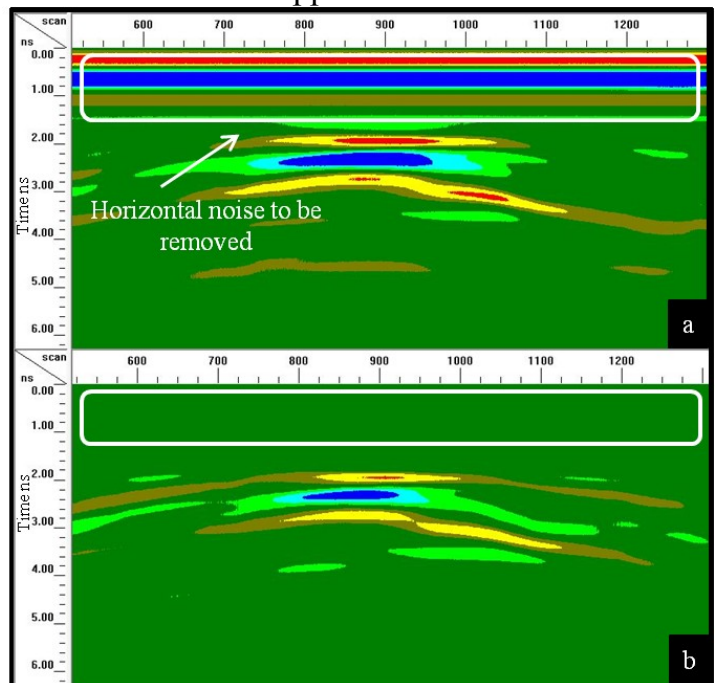

Figure 7: Portion of GPR data (1500 MHz antenna) of the wooden box buried in the sand. a, before background removal process, $\mathrm{b}$, after background removal.

The Hilbert Transform expresses the relationship between the phase and magnitude of the signal, or between its real and imaginary parts. It allows the phase of a signal to be reconstructed from its amplitude. The Hilbert Transform is used to display subtle properties of the earth. The magnitude display is useful for indicating the raw energy reflected from an object or layer. The radar wavelet itself may not always be a clear indicator of energy levels because it consists of several cycles. The instantaneous frequency indicates how the earth is filtering the radar signal (Fig. 8).

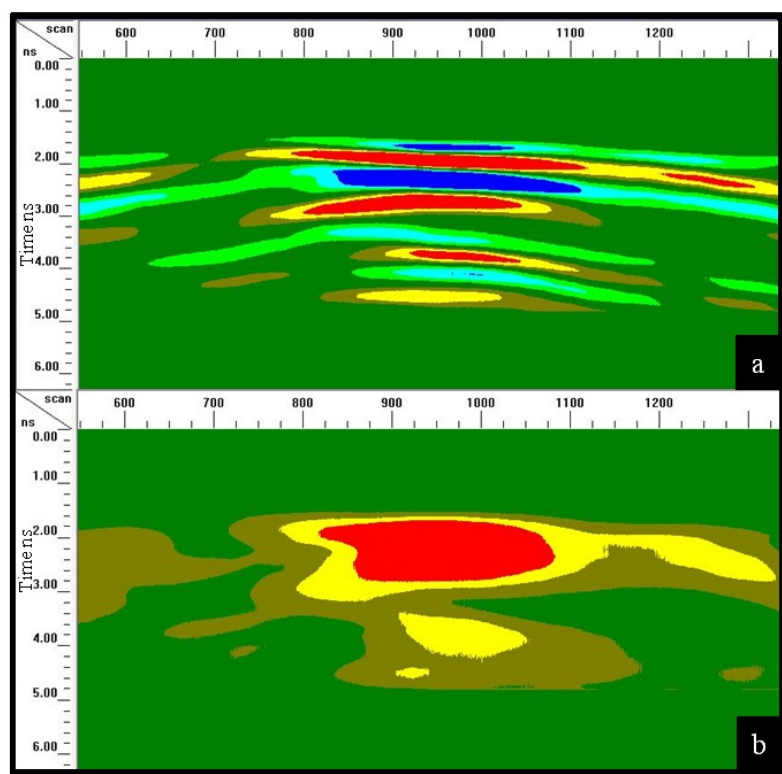

Figure 8: Portion of GPR data (1500 MHz antenna) of the plastic box buried in the sand, a, before Hilbert (Magnitude display) Transform process, b, after Hilbert Transform.

\section{Results and Discussions}

First of all, we examined two different antenna configurations to observe the effect of the survey direction in relation to the antenna radiation pattern. Fig. 9 shows the antenna's radiation pattern for different 
survey directions. Fig. 9 (a) shows the antenna configuration by the $60^{\circ}$ radiation pattern, (b, c, d, and e) shows the GPR data (for the buried fill iron, empty plastic, fill plastic and fill wooden boxes) produced with the previous configuration. Fig. 9 (f) shows the antenna configuration by the $90^{\circ}$ radiation pattern, (g, h, i, and j) shows the GPR data (for the buried fill iron, empty plastic, fill plastic and fill wooden boxes) produced with this configuration. The two configurations gave approximately the same results because of no long axis for the boxes. The configuration showed in Fig. 9 (a) gave the highest resolution for the entire buried box. For the previous reason this configuration was selected for further analysis.

For all the buried boxes, we got a clear and high reflection from the top of the buried boxes. All of the four situations (filled iron, filled plastic, filled wooden, and empty plastic boxes) can be detected with the GPR technique.

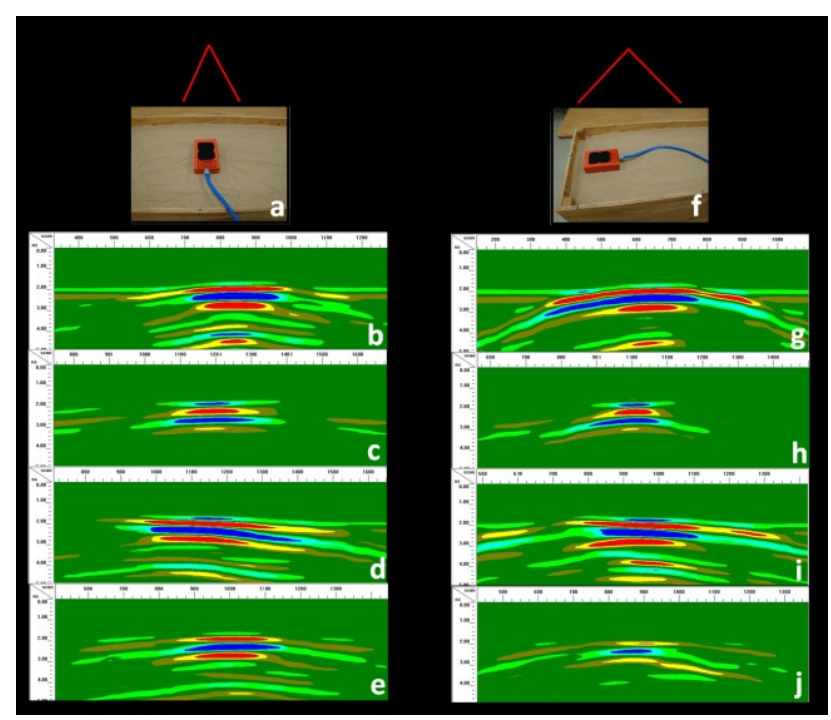

Figure 9: (a) the antenna configuration by the $60^{\circ}$ radiation pattern, (b, c, d, and e) shows the GPR data (for the

buried fill iron, empty plastic, fill plastic and fill wooden boxes) respectively produced with the previous configuration. (f) shows the antenna configuration by the $90^{\circ}$ radiation pattern, (g, h, i, and j) shows the GPR data (for the buried fill iron, empty plastic, fill plastic and fill wooden boxes) respectively produced with this configuration.

For the four cases, all the boxes are predictable and they are detected by GPR technique clearly. The reflection strength is different in these buried boxes (Fig. 10). One of the important GPR signal properties in the interpretation is the polarity of the reflected signal; it is dictated by the nature of the change in the electrical properties which cause the reflection. Reflection is caused by a change in the electromagnetic impedance. A higher impedance target yields a positive reflection coefficient whereas a lower impedance target yields a negative reflection coefficient. In GPR, metal is a very low impedance material and will always give rise to a negative (white-black-white bands) reflection wavelet. On the other hand, a void in soil or rock and the empty box represents a high impedance material and will give rise to a positive (black-white-black) reflection wavelet. The iron box has the highest reflection value about (16500) and it has the highest negative peak, its color sequence (in a gray color display) is white-black-white and the negative side has the highest reflection amplitude (Figs. 10 and 11).

The plastic (filled with iron and copper material) box has same GPR signal behavior, it has a negative wavelet (white-black-white bands) and the negative side has the highest value, but the reflection values in this case is lower than the iron box case(about 12500)(Figs. 10 and 12).

For the wooden (filled with iron and copper material) box, also the signal behavior is the same for the previous cases. The signal has a negative (white-black-white bands) wavelet and the negative side has the highest value, but the reflection values are lower than the two previous cases (about 9500) (Fig. 10).

For the empty plastic box, the case is different completely; the GPR signal has a positive ((black-whiteblack) wavelet and the wavelet positive side has the high reflection value related to the negative side. The signal has a negative (white-black-white bands) wavelet and the negative side has the highest value, but the reflection values are lower than the two previous cases (about 7200) (Fig. 10). Note the signal polarity of the four cases in Fig. 10 (A, a) shows a portion of GPR data of filled iron box, the first half cycle located in the negative side. The behavior is the same foe the filled plastic box and filled wooden box. For the empty plastic box, the polarity is positive and the first half cycle located in the positive side. Also this figure shows the strength of reflection, 
iron box has the highest reflection, the empty plastic one has the lowest one while the filled plastic and filled wooden boxes have the middle values (look at the color strength). In the gray(white and black) display The black color shows the high reflection in the negative side and white color indicates the strong reflection in the positive side while the gray color indicates no reflection and small (or zero) dielectric constant.

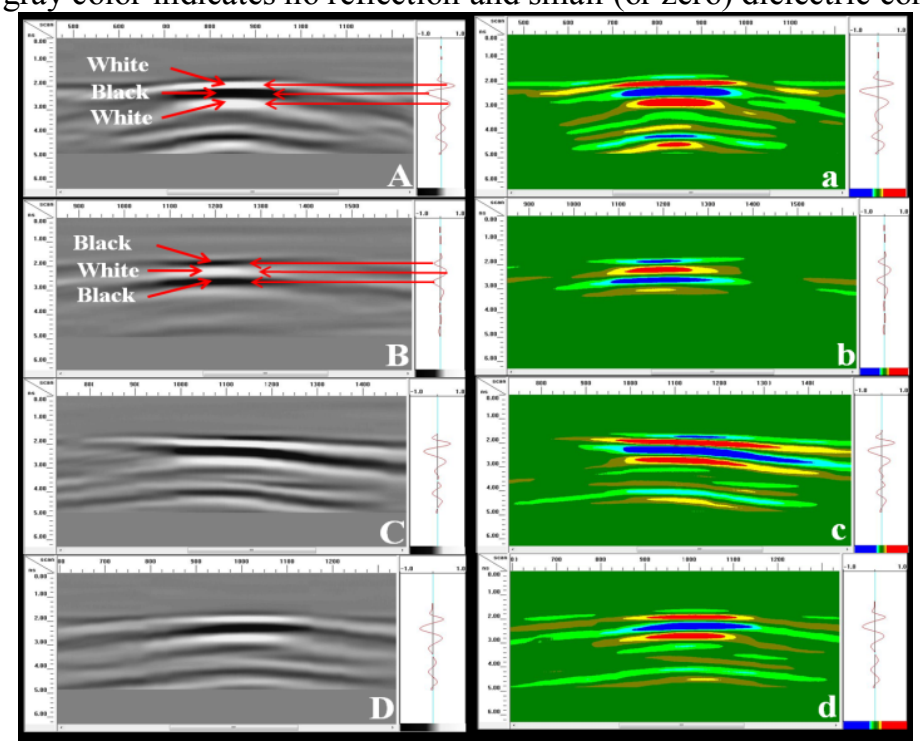

Figure 10: Portions of GPR data (1500 MHz antenna, gray and colored display) of, (A, a) the filled iron box (see the color sequences, white-black-white); (B, b) the empty plastic box (see the color sequences, black-whiteblack); (C, c) the filled plastic box, and (D, d) the filled wooden box.

For the iron filled box (filled with iron and copper materials) (Fig. 11), it gave the highest reflection. The horizontal dimensions of the box are not calculated because we use the time mode for the survey and the survey speed is hard to control therefore the horizontal dimensions will be different in each survey due to the differences in the survey speed. The target's horizontal dimensions in the outdoor (field) are so easy and accurate to measured and calculated because the distance mode will chosen and the survey wheel and another distance instruments can be used.

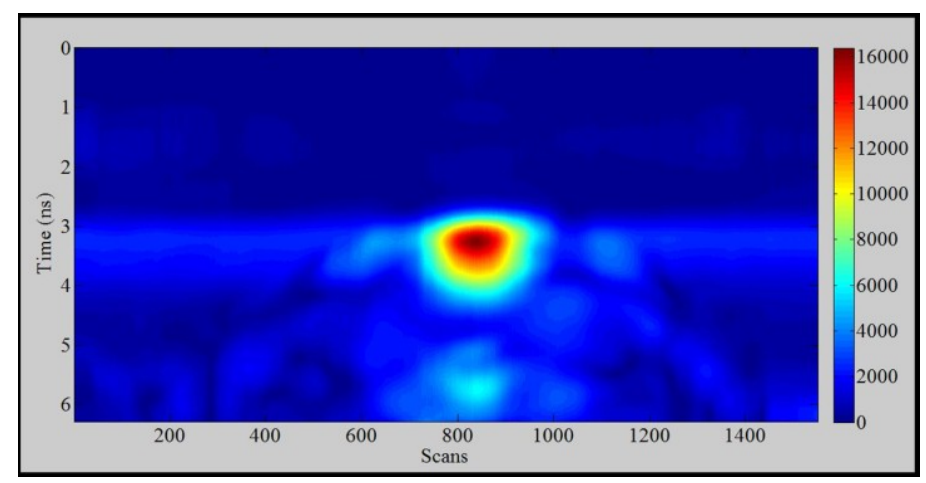

Figure 11: Processed GPR data (1500 MHz antenna) of the iron box buried in the sand after Hilbert transfer.

The thickness of the iron box can calculate by using (5):

$D=(0.3 \mathrm{~m} / \mathrm{ns} * 4.2 \mathrm{~ns}-2.7 \mathrm{~ns}) / 2 \sqrt{ } 10$

$D=0.0714 \mathrm{~m}$

This result is very close to the real high or thickness of the iron box. Also the depth to the buried object can be calculate by using the same equation, when the signal travel time and the host medium's dielectric constant are known. For the iron box, the two ways travel time is $2.7 \mathrm{~ns}$ and the sand dielectric constant is 2.8 therefore, the depth for the box is:

$D=(0.3 \mathrm{~m} / \mathrm{ns} * 2.7 \mathrm{~ns}) / 2 \sqrt{2} .8$

$D=0.251 \mathrm{~m}$

This value is very close to the depth value of the buried iron box. 
For the plastic box (filled with iron and copper materials) (Fig. 12), it gave a reflection lower than the last one. By using the same procedure, the depth to the target and the target thickness can be calculated.

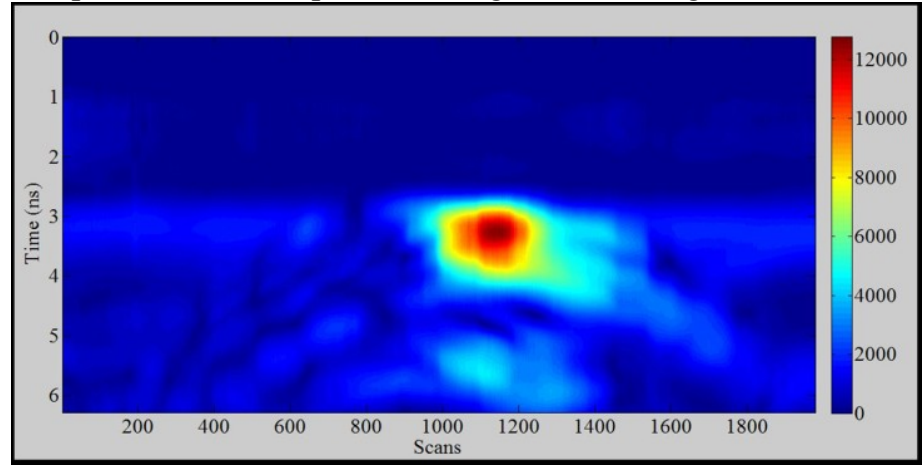

Figure 12: Processed GPR data (1500 MHz antenna) of the plastic box buried in the sand after Hilbert transfer.

The wooden box (Fig. 13) shows a reflection value lower that the iron and plastic filled reflections, while empty plastic buried box (Fig. 14) shows the lowest reflection value due to the air dielectric constant that equal to 1 . For each of these cases we can calculate the depth and thickness of the subsurface target.

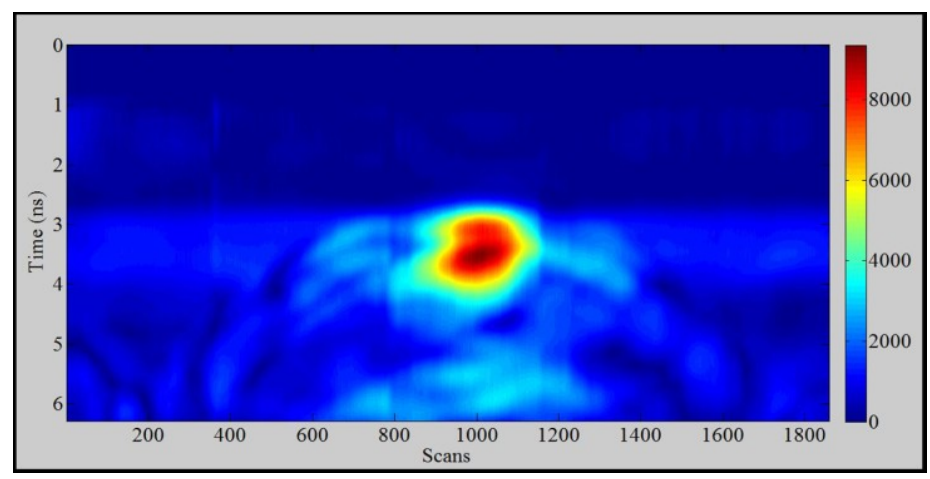

Figure 13: Processed GPR data (1500 MHz antenna) of the filled wooden box buried in the sand after Hilbert transfer.

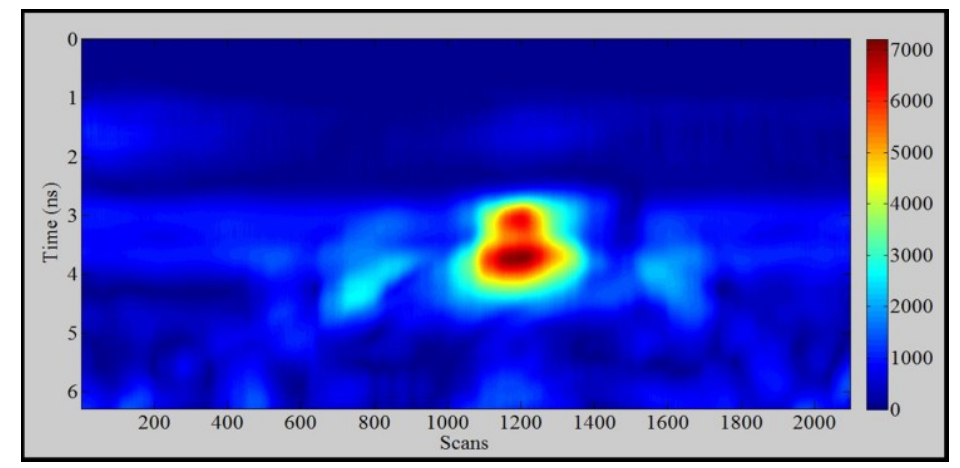

Figure 14: Processed GPR data (1500 MHz antenna) of the empty plastic box buried in the sand after Hilbert transfer.

To see the high peak s polarity and value, and the wavelet polarity, one scan for each case had been plotted. For the filled iron box (Fig. 15 (a)), scan number 847 shows that the wavelet polarity is negative (the first half cycle locate in the negative side), also the highest peak value locate in the negative side (in this case it equal $-2.129 * 10^{4}$ or -21290 ). The positive two peaks are smaller than the previous one (they are equal $1.789 * 10^{4}$ or 17890 and $1.219 * 10^{4}$ or 12190 ).

Fig. 15 (b) shows one scan cross the filled plastic box (scan number 1110). The wavelet polarity is negative (it goes down) and the high reflection peak toward the negative side $\left(-1.608^{*} 10^{4}\right.$ or -16080$)$. The other two positive peaks have a reflections lower that the negative peak (they are equal to $1.518^{*} 10^{4}$ or 15180 and 9685).

For the scan number 1600 that picked from the GPR data cross the empty plastic box (Fig. 15 (c)); it showed that the wavelet polarity is positive (it goes up in the positive side) and the high reflection peak locate in 
the positive side (2962). The other two negative peaks have a reflections lower that the positive peak (they are equal to -1921and -23383).

Finally, the scan number 1015 of the data cross the filled wooden box indicates that the polarity is negative and the highest peak locate in the negative side (-7759) while the other two positive peak have lower values than the previous one (they are equal to 5809and 3444) (Fig. 15 (d)).

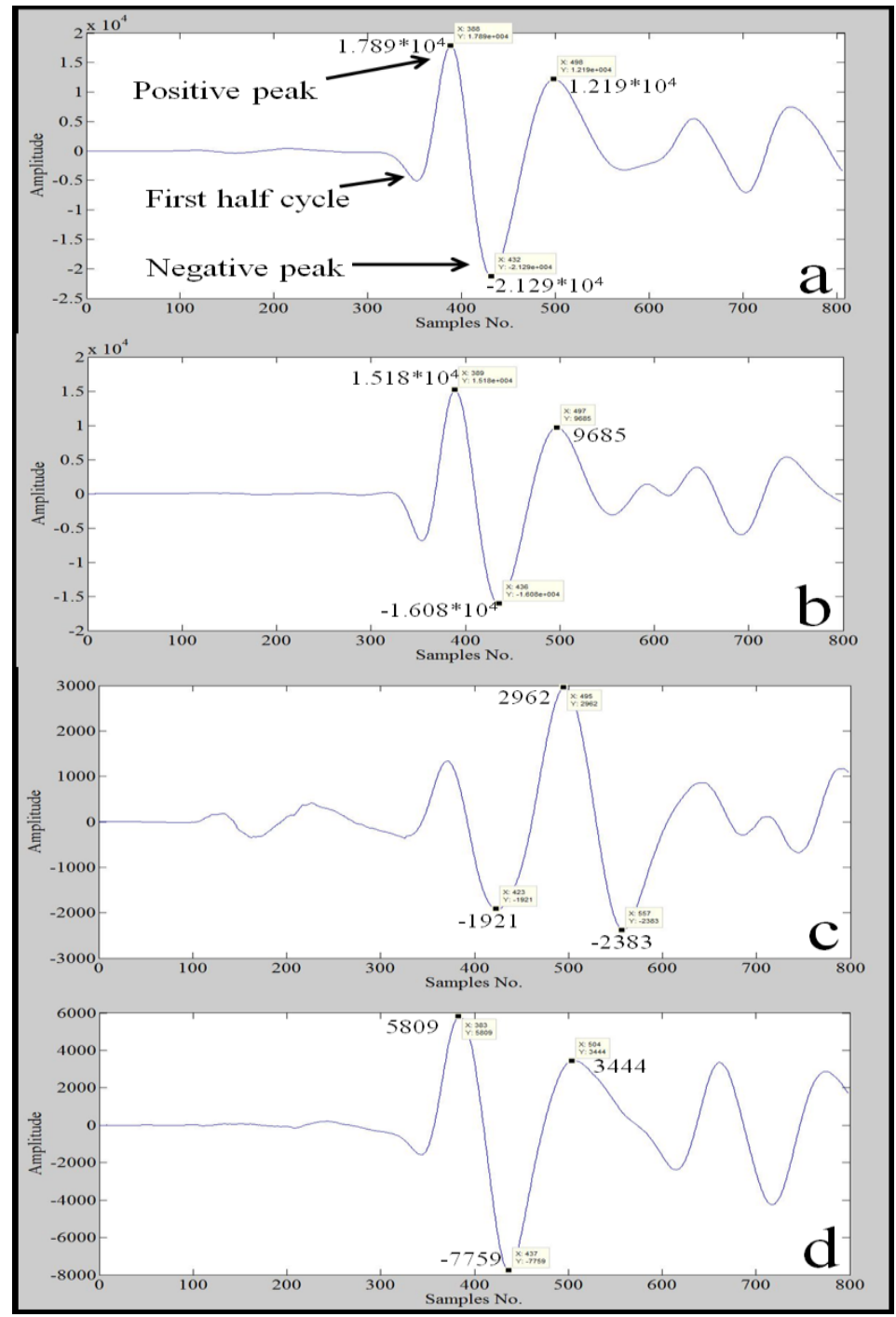

Figure 15: a, b, c, d, GPR scans cross, filled iron, filled plastic, empty plastic, and filled wooden boxes. Note the variety in the wavelet polarities and peaks reflection for the four cases.

\section{Conclusions}

The Ground Penetrating Radar is one of the best geophysical techniques to detect and image subsurface targets. One of the important target electrical properties is the dielectric constant, the object that has a high dielectric constant will be a high reflection and predictable by GPR.

Antenna's configurations (polarizations) or antenna radiation pattern do not affect the GPR signal and resolution because the targets approximately have same length axis.

For the four cases, (filled iron, filled plastic, filled wooden, and empty plastic boxes), all of them are predictable and they are detected by GPR technique clearly. Also we can calculate the targets dimensions and targets depth directly in the field. The reflection strength is different in these buried boxes. Filled Iron box has the negative wavelet polarity and has the highest reflection about (16500) and it has the highest negative peak, its color sequence (in a gray color display) is white-black-white and the negative side has the highest reflection amplitude $\left(-2.129 * 10^{4}\right)$.

Filled plastic box also has a negative wavelet polarity and has high reflection about (12000) and the high peak locate in the negative side, its color sequence (in a gray color display) is white-black-white and the negative side has the highest reflection amplitude $\left(-1.608 * 10^{4}\right)$. For the filled wooden box, the case is the same 
with the reflection lower than the previous two (9500) with a negative wavelet polarity and the highest peak (7759) is negative.

For the empty plastic box the issue is completely different, the wavelet polarity is positive and the highest peak (2962) located in the positive side also the color sequence is (black-white-black) due to the decrease in the dielectric constant. The reflection is the lowest one (7200).

Finally, based on the author's laboratory experiments results, the weapons caches and unexploded ordnance (UXO) are predictable and detectable by the GPR technique. Also we can guess the weapons containers type (wooden, plastic, and metallic containers), also the targets dimensions and depth can calculated in the field.

\section{Acknowledgements}

The authors are thankful to Dr. Haydar Al-Shukri (Prof. of Applied Science/UALR/ USA) and Dr. Hanan Mahdi (Research Associate Prof. of Graduate Institute of Technology/UALR/ USA) for their unlimited assistance.

\section{References}

[1] D.J. Daniels, D.J. Gunton, H.F. Scott, Introduction to subsurface radar, IEEE Proc., 1988, 135, 278-320.

[2] D.J. Daniels, Ground penetrating radar (2nd Edition. IET, 2004).

[3] M. Loken, Use of Ground Penetrating Radar to evaluate Minnesota roads (USA: Minnesota Department of Transportation, 2007).

[4] B. C. Lawrence, M. C. Catherine, Ground-penetrating Radar Techniques and Three-dimensional Computer Mapping in the American Southwest, Journal of Field Archaeology, 25(4), 1998, 417-430.

[5] K. Kelley, W.J. Donald, Geophysical survey as a management tool for historic properties, Society for Historical Archaeology 37 th Annual conference, St. Louis, MO, 2004.

[6] P.A. Annan, GPR methods for hydrogeological studies, in Y.Rubin, S.Hubbard (Ed.) Hydrogeophysics, New York: Springer, 2005) 413-440.

[7] J. Doolittle, B. Jenkinson, D. Hopkins, M. Ulmer, W. Tuttle, Hydropedological investigations with ground-penetrating radar (GPR): Estimating water-table depths and local ground-water flow pattern in areas of coarse-textured soils, Geoderma, 131(3-4), 2006, 317329.

[8] T. Saarenketo, T. Scullion, Roadevaluation with groundpenetrating radar, Journal of Applied Geophysics, 43(2-4), 2000, 119-138.

[9] C. Yuejian, D. Shongtao, F.L. Joseph, P. John, Implementation of Ground Penetrating Radar (Final report, Minnesota Department of Transportation, 2007).

[10] A. K. Benson, Applications of ground penetrating radar in assessing some geological hazards: examples of groundwater contamination, faults, cavities, Journal of Applied Geophysics, 33(1-3), 1995, 177-193.

[11] H.K. Chlaib, M. Su, N. Abd, A. Catakli, H. Mahdi, H. Al-Shukri, Detection of weakness zones in Helena levee using Ground Penetrating Radar, United States Society on Dams (USSD) 2014 Annual Meeting and Conference, San Francisco, CA, 2014.

[12] H.K. Chlaib, H. Mahdi, H. Al-Shukri, M. Su, A. Catakli, N. Abd, Using ground penetrating radar in levee assessment to detect small scale animal burrows, Journal of Applied Geophysics, 103, 2014, 121-131.

[13] H.K. Chlaib, H. Mahdi, H. Al-Shukri, M. Su, A. Catakli, N. Abd, Levee evaluation with Ground-Penetrating Radar, United States Society on Dams (USSD) 2013 Annual Meeting and Conference, Phoenix, AZ, 2013, 30-45.

[14] H.M. Jol, Ground penetrating radar: theory and applications (First edition: Elsevier, 2009).

[15] Geophysical Survey Systems Inc., SIR System-3000 User's manual (Geophysical Survey Systems Inc., North Salem, New Hampshire, 2011).

[16] Stevens ${ }^{\circledR}$ Water Monitoring System Inc., POGO quick start instructions (Steven's Water Monitoring, 2007).

[17] Geophysical Survey Systems Inc., RADAN 6.6 user's manual (Geophysical Survey Systems Inc., North Salem, New Hampshire, 2009).

[18] Geophysical Survey Systems Inc., RADAN 7 user's manual (Geophysical Survey Systems Inc., North Salem, New Hampshire, 2011) 